\title{
Undifferentiated carcinoma with osteoclast- like giant cells of the pancreas harboring KRAS and BRCA mutations: case report and whole exome sequencing analysis
}

\author{
Guangjian Yang ${ }^{1}$, Jiangxia Yin², Kai Ou', Qiang Dư ${ }^{3}$, Wenhao Ren ${ }^{3}$, Yujing $\mathrm{Jin}^{4}$, Liming Peng ${ }^{1}$ and Lin Yang ${ }^{1^{*}}$ (D)
}

\begin{abstract}
Background: Undifferentiated carcinoma with osteoclast-like giant cells (UC-OGC) is an extremely uncommon pancreatic neoplasm that comprises less than $1 \%$ of all exocrine pancreatic tumors. To date, cases and data from whole-exome sequencing (WES) analysis have been reported by specific studies. We report a case of pancreatic UCOGC with a literature review, and provide novel insights into the molecular characteristics of this tumor entity.

Case presentation: A 31-year-old male presented with intermittent abdominal pain for several months, and positron emission tomography (PET) showed isolated high metabolic nodules during the pancreatic uncinate process that were likely to be malignant disease. Pathological examination after radical excision revealed UC-OGC associated with poorly differentiated adenocarcinoma at the head of the pancreas. The disease recurred 7.4 months after radical surgery. The KRAS p.G12D (c.35G > A) and somatic BRCA2 p.R2896C (c.8686C > T) mutations were detected by subsequent WES analysis. The patient showed no response to platinum-based systemic chemotherapy, and his condition quickly worsened. He finally died, with an overall survival of 1 year.

Conclusions: As an extremely uncommon tumor entity, UC-OGC is really a unique variant of conventional pancreatic ductal adenocarcinoma due to its similarities, as shown by genomic WES analysis. Clinical examination and molecular analysis by WES could further indicate potential treatment strategies for UC-OGC.
\end{abstract}

Keywords: Undifferentiated carcinoma with osteoclast-like giant cells, Pancreatic ductal adenocarcinoma, Pancreas, Case report, Whole exome sequencing

\section{Background}

Pancreatic cancer is the thirteenth most malignancy worldwide [1], with a high mortality that is equal to the incidence. Pancreatic ductal adenocarcinoma (PDAC), as the most common pathologic type, is associated with poor treatment response and poor prognosis. The

\footnotetext{
* Correspondence: linyangcicams@126.com

'Department of Medical Oncology, National Cancer Center/National Clinical Research Center for Cancer/ Cancer Hospital, Chinese Academy of Medical Sciences and Peking Union Medical College, Beijing 100021, China Full list of author information is available at the end of the article
}

reported evidence has revealed that the molecular characteristics of PDAC include alterations in the driver gene KRAS and the tumor suppressor genes TP53, CDKN2A and SMAD4 [2-6]. Undifferentiated carcinoma with osteoclast-like giant cells (UC-OGC), as a variant of anaplastic carcinoma of the pancreas, is observed extremely rarely in clinical practice $[7,8]$. Worldwide, sporadic case reports have indicated that UC-OGC comprises less than $1 \%$ of all exocrine pancreatic tumors $[9,10]$. Analysis of 38 UC-OGC cases demonstrated that it showed a better clinical course compared with that of

(c) The Author(s). 2020 Open Access This article is licensed under a Creative Commons Attribution 4.0 International License, which permits use, sharing, adaptation, distribution and reproduction in any medium or format, as long as you give appropriate credit to the original author(s) and the source, provide a link to the Creative Commons licence, and indicate if changes were made. The images or other third party material in this article are included in the article's Creative Commons licence, unless indicated otherwise in a credit line to the material. If material is not included in the article's Creative Commons licence and your intended use is not permitted by statutory regulation or exceeds the permitted use, you will need to obtain permission directly from the copyright holder. To view a copy of this licence, visit http://creativecommons.org/licenses/by/4.0/. The Creative Commons Public Domain Dedication waiver (http://creativecommons.org/publicdomain/zero/1.0/) applies to the data made available in this article, unless otherwise stated in a credit line to the data. 
conventional PDAC [8]. In addition, a few molecular studies of UC-OGC reported that KRAS mutations most frequently occurred, which was similar to that observed in PDAC [11-13]. Additionally, one detailed study reported the molecular features of UC-OGC by performing whole-exome sequencing (WES) analysis [14], and all these results implied that pancreatic UC-OGC was analogous to PDAC. To date, more cohorts of patients are needed to investigate the pathological and genetic features of this unique tumor variant. Herein, we report a case of pancreatic UC-OGC harboring the KRAS p.G12D mutation and somatic BRCA2 mutation, as detected by WES, in a patient experienced reduced disease-free survival (DFS) and overall survival (OS). Furthermore, we provide a literature review of UC-OGC studies and analyze them to obtain novel insights regarding the molecular characteristics of this tumor entity.

\section{Case presentation}

A 31-year-old male with no past medical or family history of disease presented with intermittent abdominal pain lasting almost 2 months, and he was admitted to the local hospital on February 28, 2017. Positron emission tomography (PET) showed isolated high metabolic nodules during the pancreatic uncinate process that were likely to represent malignant disease (Fig.1a, b).
The patient then underwent radical pancreaticoduodenectomy on March 9, 2017. Pathological examination after radical excision showed poorly differentiated ductal adenocarcinoma associated with UC-OGC at the head of the pancreas (Fig.2a-d). Immunohistochemistry staining revealed that the cells were positive for CD68 and CK7, whereas the cells were negative for vimentin and S-100 (Fig. 2e, f). The tumor was measured to be $3 \times 3 \times 2 \mathrm{~cm}$ in size and exhibited invasion of the nerves, nearby pancreatic tissues, duodenum and the lower part of the common bile duct. The surgical margins were negative, and there was no discovery of lymph node metastasis. The surgical-pathological staging of the tumor was IIA (T3NOM0) according to the 7th edition of the American Joint Committee on Cancer (AJCC)/Union for International Cancer Control (UICC) TNM staging system.

Adjuvant chemotherapy with gemcitabine and albuminbound paclitaxel was administered starting on April 10, 2017 for six cycles, and the toxicity was acceptable. However, the patient developed a backache 2 months after the termination of adjuvant chemotherapy. The contrasted computed tomography (CT) scan performed on November 27, 2017 showed multiple lymph node metastases in the mesenteric region (Fig. 1c) and peritoneum (Fig. 1d) with a serum CA199 level $>900 \mathrm{U} / \mathrm{ml}$. Exploratory laparotomy was performed on November 29, 2017, and affirmed peritoneal metastasis was confirmed by peritoneal biopsy. The patient afterwards received systemic
A

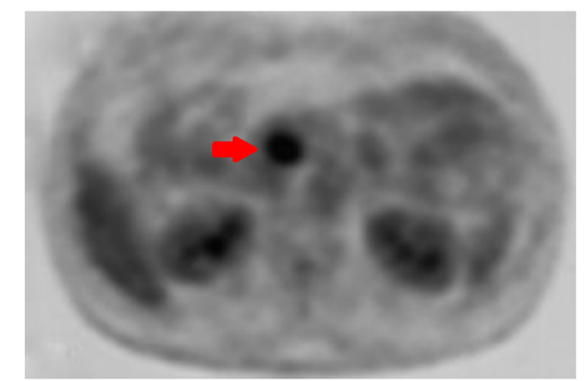

C

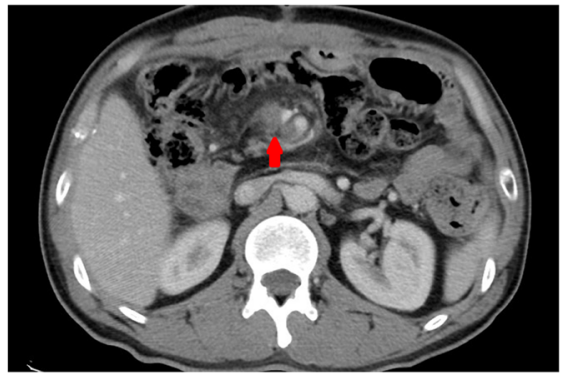

B

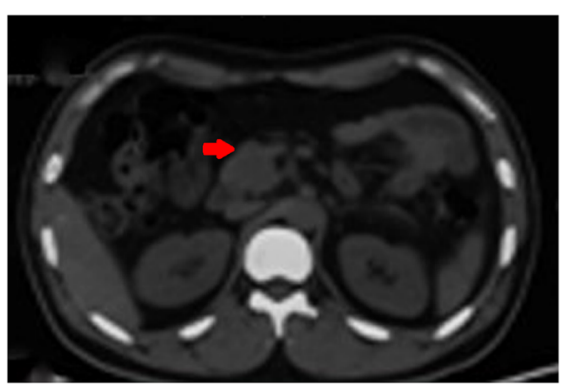

D

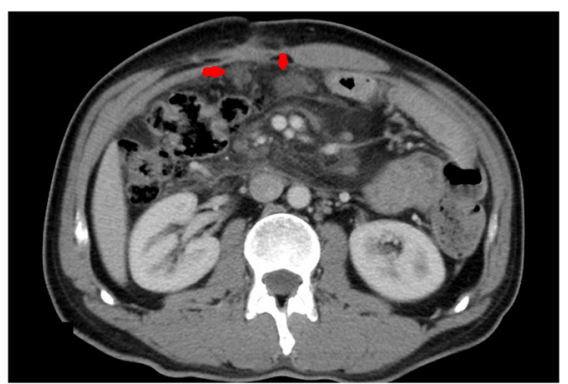

Fig. 1 The PET showed high metabolic nodules at pancreatic uncinated process and inclined to be malignant disease at baseline (a, b). The contrasted CT scan showed multiple lymphatic metastases in the mesenteric region(c) and peritoneum (d) beyond termination of adjuvant chemotherapy 

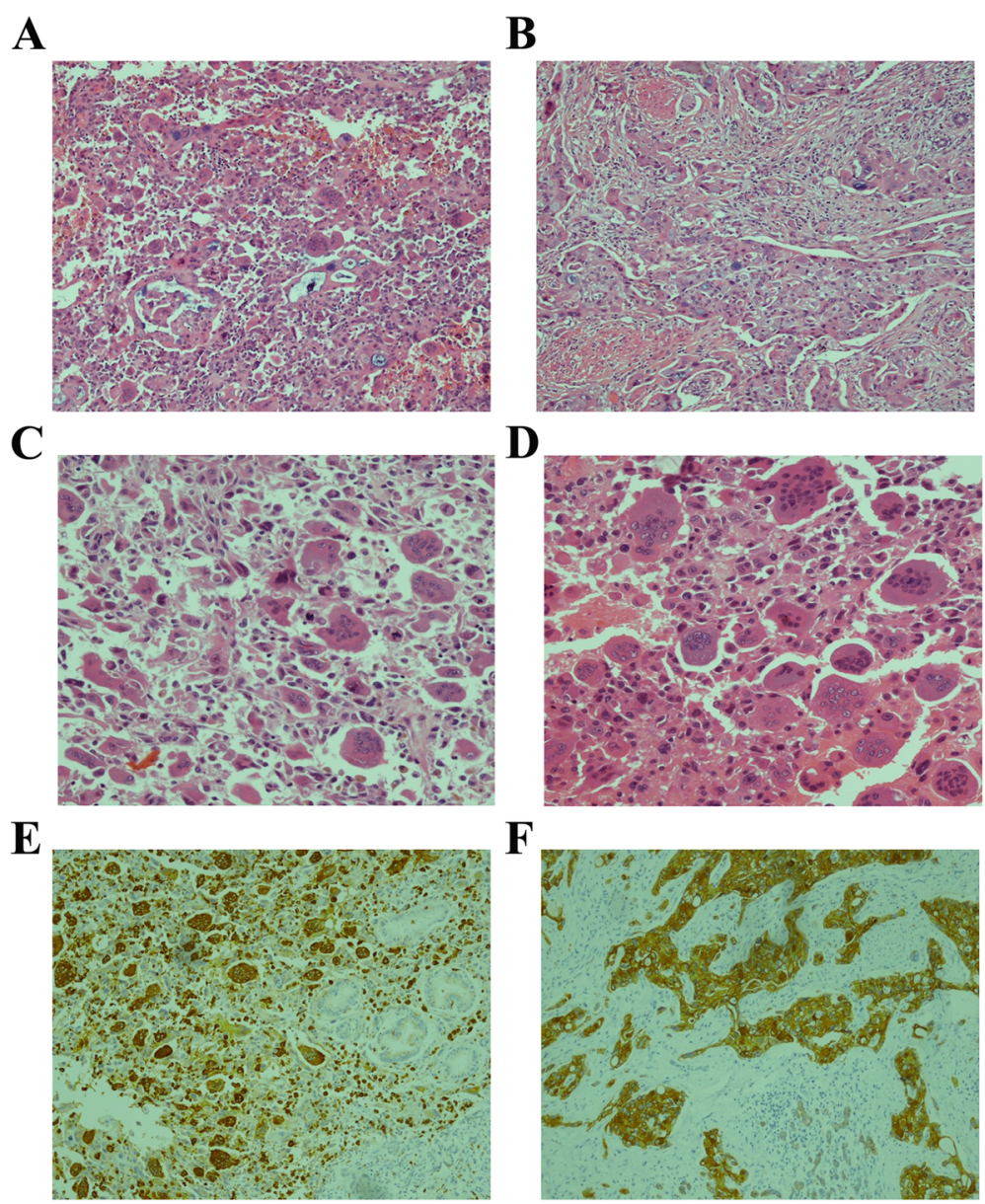

Fig. 2 Histological features of pancreatic undifferentiated carcinoma with osteoclast-like giant cells (UC-OGC) under 100X (H\&E, a). UC-OGC associated with poorly differentiated ductal adenocarcinoma component under 100X (H\&E, b). The UC-OGC composed of anaplastic carcinoma and intermixed with pleomorphic neoplastic mononuclear cells and multinucleated osteoclast-like giant cells under 200X (H\&E, c and d).

Osteoclast-like giant cells of the tumor were stained positive for CD68 (IHC, e). Staining was positive for CK7 diffusely in the PDAC component of the tumor $(I H C, \mathbf{f})$

chemotherapy with the FOLFIRINOX regimen (combination of oxaliplatin, irinotecan, fluorouracil and leucovorin) for two cycles. Unfortunately, the serum tumor marker CA199 level was elevated to $1595 \mathrm{U} / \mathrm{ml}$ after two treatment cycles, and the patient's condition deteriorated due to obvious myelosuppression and digestive tract toxicity caused by the chemotherapeutic drugs. Finally, he had to suspend chemotherapy and was admitted to our hospital on January 11, 2018.

WES analysis was performed, and the KRAS p. G12D (c. $35 \mathrm{G}>\mathrm{A}$ ) and somatic $B R C A 2$ p. R2896C (c. $8686 \mathrm{C}>$ T) mutations were detected in both surgical formalinfixed paraffin-embedded (FFPE) tumor tissues and plasma ctDNA samples. Additionally, WES indicated that the tumor did not show microsatellite instability (MSI) and did not present a high tumor mutational burden (TMB). Considering the poor condition of the patient and the fact that the polyadenosine diphosphate- ribose polymerase (PARP) inhibitor olaparib was not available, we administered apatinib combined with tegafur/gimeracil/oteracil potassium capsules (S-1) for his disease. However, the patient's condition worsened rapidly with the occurrence of fever, jaundice and vomiting after 1 month of treatment with this regimen, and eventually he died on March 12, 2018. The disease-free survival (DFS), which was defined as the time from radical surgery to disease recurrence, was just 7.4 months. The overall survival (OS), which was defined as the time between the primary diagnosis of UC-OGC and death, was only 12.6 months.

\section{Discussion and conclusion}

Undifferentiated carcinoma of the pancreas, is a highly malignant tumor that tends to exhibit invasion of the perineum, lymph nodes and blood vessels and is called "giant cell carcinoma" or "pleomorphic large cell 
carcinoma" [15]. Tumors with osteoclast-like giant cells (OGCs) have been documented in a variety of organs, including the kidney, breast, thyroid gland, heart, parotid gland and skin [7, 16-18]. The UC-OGC is composed of pleomorphic neoplastic mononuclear cells that are and intermixed with large non-neoplastic multinucleated giant cells, as observed under microscopy [19], and it is suggested that UC-OGC is derived from epithelial tumors and the components of vimentin-positive carcinoma, which represent the mesenchymal transition of ductal cells [20,21]. Based on the pathological features, the World Health Organization (WHO) had classified UC-OGC as a unique PDAC variant in 2010 [22].

The OGCs within the background of anaplastic malignant cells in UC-OGC are commonly considered to be of benign histiocytic origin, which has been supported in several cases by their immunoreactivity with CD68 [16]. Currently, it is hypothesized that OGC recruitment is a result of chemotactic factors produced by neoplastic cells and is indicative of a better prognosis [16]. Notably, such tumors can be classified as pure UC-OGC if they are not associated with a distinct neoplasm with a different morphology [14]. Luchini et al. [14] reported that the median OS (mOS) of 16 analyzed UC-OGC patients was 20 months, and the mOS of patients with pure UCOGC was significantly higher than that of patients with associated PDAC (36 vs. 15 months, $P=0.04$ ). Furthermore, it revealed an UC-OGC associated with PDAC conferred a five-fold increased risk of death [14], which was in accordance with the survival data reported by Muraki et al. [8]. The presence of UC-OGC in our case was confirmed by CD68 staining in the margin of undifferentiated tumors, and immunoreactivity with CK7 showed the presence of an associated adenocarcinoma component, which proved that this particular case was not pure UC-OGC. The 31-year-old male patient in our case survived for only 1 year, which was similar to the length of survival previously reported above $[8,14]$.

WES analysis of $8 \mathrm{UC}-\mathrm{OGC}$ patients had revealed that KRAS oncogenic mutations were identified in all analyzed cases, which implied that this tumor entity shared similar genomic features with conventional PDAC [14]. In addition, other previous studies also indicated the prevalence of KRAS mutations in UC-OGC [11-13, 23]. Based on the WES outcome for the UC-OGC cohort reported by Luchini et al. [14], all variants of KRAS mutations were found in codon 12, including the G12V, G12D and G12R mutations. In addition, additional somatic mutations in the tumor suppressor genes TP53, CDKN2A and SMAD4 were detected in these UC-OGC cases, which further indicated that UC-OGC is a unique phenotype of PDAC due to the fact that these alterations either commonly appear in PDAC [14]. Additionally, Luchini et al. found the same SERPINA3 variant
(p.M290L) in a hotspot region in two UC-OGC cases and suggested that it may be an oncogene that had been previously reported in squamous cell carcinoma in the cervix [14]. SERPINA3 encodes $\alpha$-1-antichymotrypsin, which inhibits a plasma protease belonging to the serine protease inhibitor class [24]. Of note, the upregulation of SERPINA3 is correlated with increases in cancer cell migration and invasion, and indicated a poor prognosis for several cancer types [25, 26]. WES analysis also suggested that GLI3 was a driver gene of UC-OGC, as it was detected in two cases [14]. GLI3, as a target of microRNAs and transcription factors of the Hedgehog signalling pathway, is known to be upregulated in multiple cancers, in which it results in cancerous cell behaviour such as anchorage-independent growth, angiogenesis, proliferation and migration [27]. Except for the above mutations, it was difficult to interpret the importance of the other nonsynonymous mutations in MEGF8, MAGEB4 and TTN detected by WES [14]. Muller et al. reported that the dosage gain in KRAS p. G12D dosage gain was not only related to early tumor progression, but also associated with metastasis in PDAC [28]. Unfortunately, there is currently no highly selective agent to suppress KRAS-mutated cancer. The WES analysis of our case indicated that the KRAS p. G12D mutation functioned as a major driver that resulted in the activation of downstream signalling pathways and high-grade disease malignancy. The patient suffered a pancreatic tumor at a young age and his disease progressed rapidly within an extremely short time after the previous radical operation. These results indicated that KRAS mutations in both in UC-OGC and PDAC result in the activation of oncogenes, which results in a poor prognosis, and that targeted agents against KRAS oncogenic mutations are urgently needed.

PDAC has been reported to have an immunosuppressive tumor microenvironment with a high programmed cell death-ligand 1 (PD-L1) expression, and in turn, the overexpression of PD-L1 inhibited the cytotoxic effects of activated T-cells [29]. Several studies have indicated that all indicated PD-L1 expression in PDAC is associated with a significantly poorer prognosis compared to that in patients without PD-L1 expression [29-34]. Luchini et al. investigated the PD-L1 expression patterns in pancreatic UC-OGC and finally found that PD-L1 was more frequently expressed in cases associated with PDAC than in cases associated with pure UC-OGC $(P=$ 0.04), and PD-L1-positive UG-OGC was associated with a three-fold $(P=0.034)$ higher risk of mortality than PDL1-negative UC-OGC [35]. In addition, the mismatch repair (MMR) system plays a crucial role in the repair of DNA sequence mismatches during replication. Defects in the MMR system (dMMR) could lead to errors in DNA replication, resulting in a high-TMB or increased 
MSI [36]. Thus, a high neoantigen load that increases proinflammatory cytokine levels and the activation of $\mathrm{T}$ cells is accumulated due to somatic mutations and contributes to the immunogenicity of MSI tumors with a sensitivity to immune checkpoint blockade [37]. Nevertheless, the prevalence of MSI/dMMR in PDAC is likely to be much lower than that in other gastrointestinal cancers, with only a $0-0.8 \%$ prevalence rate, as previously reported [38, 39]. Salem et al. analyzed 870 PDAC cases and found a low prevalence (1.4\%) of high TMB in PDAC, and the majority of cases had a low TMB in either MSI-high or MSI-low patients [40]. A genomic profile analysis with a large sample size including 3594 PDAC cases [6] demonstrated that MSI-high and/or TMB-high status was detected in only $0.5 \%$ of samples [6]. In addition, KRAS, TP53, CDKN2A and SMAD4 were the most frequently altered genes, and $K R A S$ mutations ranked the first, with a prevalence of $88 \%$. Additionally, alterations of the BRCA and FANC genes, which encode DNA damage repair proteins, were found in $14 \%$ of PDAC cases [6]. The tumor did not show MSI and did not present a high-TMB in our case, and the PD-L1 expression of this case was unknown. Based on the description given above, the patient associated with our case had no indication for immunotherapy.

In addition to the common KRAS oncogenic mutations, additional somatic BRCA2 alterations were detected by WES in this case. Pancreatic cancer was reported to be the third most common cancer associated with BRCA mutations [41]. Approximately 7\% of patients with pancreatic cancer carried germline mutations in $B R C A 1 / 2$, and the frequency of $B R C A 1 / 2$ mutation carriers was estimated to be at 4.9 to $26 \%$ in familial pancreatic cancer [42]. To date, the largest reported PDAC case series involving patients with germline $B R C A$ mutations showed that the median OS was 27.6 months [43]. Ashkenazi Jews have been the population with the highest prevalence of BRCA1/2 mutations in pancreatic cancer, with approximately $96 \%$ of patients having mutations in BRCA1/2 (BRCA1 185delAG, BRCA1 5382insC, or BRCA2 6174delT), and the BRCA2 6174delT variant is the most common variant in familial pancreatic cancer [44]. The PARP inhibitor olaparib had an objective response rate (ORR) of $21.7 \%$ in heavily pretreated pancreatic cancer patients with germline $B R C A 1 / 2$ mutations in a phase II study [45]. A randomized phase III study [46] showed that after first-line platinum-based chemotherapy, olaparib functioned as a maintenance therapy in pancreatic cancer patients with germline $B R C A 1 / 2 \mathrm{mu}$ tations and significantly prolonged the median PFS compared with that in patients subjected to maintenance with a placebo ( 7.4 vs. 3.8 months, $P=0.004)$.

Advances in pancreatic cancer are lacking, as it is actually a highly heterogeneous disease resistant to conventional cytotoxic chemotherapeutic drugs or targeted agents [47]. The chemotherapy regimen of FOLFIRINOX (combination of oxaliplatin, irinotecan, fluorouracil and leucovorin) [48] or gemcitabine plus albumin-bound paclitaxel [49] is the preferred first-line recommendation for the treatment of in metastatic PDAC. Some evidence has also shown that BRCA-deficient cells are more susceptible to platinum than $B R C A$ proficient cells $[50,51]$, which has been supported by several clinical trials $[52,53]$. The new version of the National Comprehensive Cancer Network (NCCN) Guidelines had recommended gemcitabine/cisplatin chemotherapy as one of the first-line regimens for BRCA1/BRCA2-mutated PDAC [54]. Waddell et al. reported that 4 patients with unstable genomes or a high $B R C A$ mutational signature burden had robust complete or partial responses to platinum-based chemotherapy among 8 PDAC patients who received the same regimen, while 3 patients without these characteristics did not respond. Subsequent research also indicated that BRCA2mutant patient-derived xenografts (PDXs) responded to cisplatin, and PDXs without mutations in a BRCA pathway gene failed to respond to cisplatin as well [55]. All these findings demonstrated that mutations in $B R C A$ pathway genes or genomic instability had potential implications for the selection of PDAC treatment. In our case, the patient was a carrier of the somatic BRCA2 mutant (p. R2896C), which has not been characterized to have known functional consequences. Subsequent bioinformatics analysis with various prediction software packages predicted the $B R C A 2 \mathrm{p}$. R2896C mutation to be neutral. The disease in this patient rapidly progressed after only two cycles of platinum-based chemotherapy, and treatment with a PARP inhibitor was not possible owing to the presence of a non-germline BRCA2 mutation.

Based on the mutational landscape of the genomics by WES, Waddell et al. [55] classified PDAC into four subtypes based on potential clinical utility according to exome and copy number variation (CNV) analyses including stable, locally rearranged, scattered and unstable. In the stable subtype, tumor genomes showed evidence of $\leq 50$ structural variations that were located randomly throughout the genome. The locally rearranged type, it exhibited at least 50 focal variations on one or two chromosomes and nearly $1 / 3$ the tumors of this subtype contained regions of copy number gain that harbored certain oncogenes. The scattered subtype exhibited nonrandom chromosomal damage and fewer than 200 structural variations. The unstable subtype exhibited a large number of structural variations (>200), and the high level of genomic instability suggested defects in DNA maintenance and potentially showed sensitivity to DNA-damaging agents. In addition, Bailey et al. 
defined pancreatic cancer according to another four subtypes, including squamous, pancreatic progenitor, immunogenic and aberrantly differentiated endocrine exocrine [5]. These different types are associated with distinct histopathological characteristics, and each inferred the presence of different mechanisms of the molecular evolution of pancreatic cancer. To some degree, the assessment of the subtype can guide accurate therapeutic selection for pancreatic cancer. Furthermore, researchers have identified five new susceptibility loci for pancreatic cancer in the Chinese population to provide effective markers for the early screening and diagnosis of this very malignant cancer [56]. In this case, WES analysis revealed that the CNV in the SOX9 gene gained approximately $1.11 \%$ variarion, whereas the $\mathrm{CNV}$ results for the KRAS and BRCA2 genes were normal. Based on the mutational landscape of pancreatic cancer illustrated above, the case in this study deserved to be classified as the stable subtype owing to the presence of less than 50 structural variation events in the CNV.

In conclusion, although pancreatic UC-OGC is extremely uncommon and complex, the current evidence has clarified that it is a unique variant of conventional PDAC due to the genomic similarities between it and PDAC revealed by WES analysis. Assessment of the clinical and molecular characteristics by WES would further provide potential treatment strategies for this tumor entity.

\begin{abstract}
Abbreviations
AJCC/UICC: American Joint Committee on Cancer/Union for International Cancer Control; CNV: Copy number variation; CT: Computed tomography; DFS: Disease-free survival; dMMR: Defects in mismatch repair system; MMR: Mismatch repair; mOS: Median overall survival; MSI: Microsatellite instability; NCCN: National Comprehensive Cancer Network; ORR: Objective response rate; OS: Overall survival; PARP: Poly adenosine diphosphate-ribose polymerase; PDAC: Pancreatic ductal adenocarcinoma; PD-L1: Programmed cell death-ligand 1; PDX: Patient-derived xenografts; PET: Positron emission tomography; S-1: Tegafur gimeracil oteracil potassium capsules; TMB: Tumor mutational burden; UC-OGC: Undifferentiated carcinoma with osteoclast-like giant cells; WES: Whole-exome sequencing; WHO: World Health Organization
\end{abstract}

\section{Acknowledgements}

We would like to take the opportunity to thank the patient and his family and all of the case co-investigators involved in the case. We would also like to give many thanks for the clinical whole exome sequencing performed by the GenomiCare Company and the professional guidance and advice it provided for this article.

\section{Authors' contributions}

GJY planned and wrote the paper; JXY provided the case; YJJ performed the imaging diagnosis; QD and WHR performed the pathological examinations; $\mathrm{KO}, \mathrm{LMP}$ and $\mathrm{LY}$ provided careful guidance on the revision of the final draft. All authors read and approved the final manuscript.

\section{Funding}

Not applicable.

\section{Availability of data and materials}

To protect the patient's privacy, the data for the current case were not allowed to be shared, which was requested by the patient in the written application.

\section{Ethics approval and consent to participate}

All procedures performed in this report were performed in accordance with the ethical standards of the institutional and/or national research committee and with the 1964 Helsinki Declaration and its later amendments or comparable ethical standards.

\section{Consent for publication}

Written consent for publication was obtained from the patient when he was alive.

\section{Competing interests}

The authors declare that they have no competing interests.

\section{Author details}

${ }^{1}$ Department of Medical Oncology, National Cancer Center/National Clinical Research Center for Cancer/ Cancer Hospital, Chinese Academy of Medical Sciences and Peking Union Medical College, Beijing 100021, China. ${ }^{2}$ Department of Oncology, Shouguang Hospital of Traditional Chinese Medicine, Weifang 262700, China. ${ }^{3}$ Department of Pathology, National Cancer Center/National Clinical Research Center for Cancer/ Cancer Hospital, Chinese Academy of Medical Sciences and Peking Union Medical College, Beijing 100021, China. ${ }^{4}$ Department of Medical Imaging, National Cancer Center/National Clinical Research Center for Cancer/ Cancer Hospital, Chinese Academy of Medical Sciences and Peking Union Medical College, Beijing 100021, China.

Received: 23 January 2020 Accepted: 17 June 2020

Published online: 26 June 2020

\section{References}

1. Siegel RL, Miller KD, Jemal A. Cancer statistics, 2017. CA Cancer J Clin. 2017; 67(1):7-30.

2. Yachida S, White CM, Naito Y, Zhong Y, Brosnan JA, Macgregor-Das AM, et al. Clinical significance of the genetic landscape of pancreatic cancer and implications for identification of potential long-term survivors. Clin Cancer Res. 2012;18(22):6339-47.

3. Jones S, Zhang X, Parsons DW, Lin JC, Leary RJ, Angenendt P, et al. Core signaling pathways in human pancreatic cancers revealed by global genomic analyses. Science. 2008;321(5897):1801-6.

4. Biankin AV, Waddell N, Kassahn KS, Gingras MC, Muthuswamy LB, Johns AL, et al. Pancreatic cancer genomes reveal aberrations in axon guidance pathway genes. Nature. 2012;491 (7424):399-405.

5. Bailey P, Chang DK, Nones K, Johns AL, Patch AM, Gingras MC, et al. Genomic analyses identify molecular subtypes of pancreatic cancer. Nature. 2016;531(7592):47-52.

6. Singhi AD, George B, Greenbowe JR, Chung J, Suh J, Maitra A, et al. Real-time targeted genome profile analysis of pancreatic ductal adenocarcinomas identifies genetic alterations that might be targeted with existing drugs or used as biomarkers. Gastroenterology. 2019;156(8):2242-53.

7. Loya AC, Ratnakar KS, Shastry RA. Combined osteoclastic giant cell and pleomorphic giant cell tumor of the pancreas: a rarity. An immunohistochemical analysis and review of the literature. JOP. 2004;5(4): 220-4.

8. Muraki T, Reid MD, Basturk O, Jang KT, Bedolla G, Bagci P, et al. Undifferentiated carcinoma with osteoclastic giant cells of the pancreas: clinicopathologic analysis of 38 cases highlights a more protracted clinical course than currently appreciated. Am J Surg Pathol. 2016;40(9):1203-16.

9. Jo S. Huge undifferentiated carcinoma of the pancreas with osteoclast-like giant cells. World I Gastroenterol. 2014;20(10):2725-30.

10. Molberg KH, Heffess C, Delgado R, Albores-Saavedra J. Undifferentiated carcinoma with osteoclast-like giant cells of the pancreas and periampullary region. Cancer. 1998;82(7):1279-87.

11. Westra WH, Sturm P, Drillenburg P, Choti MA, Klimstra DS, Albores-Saavedra $\mathrm{J}$, et al. K-ras oncogene mutations in osteoclast-like giant cell tumors of the pancreas and liver: genetic evidence to support origin from the duct epithelium. Am J Surg Pathol. 1998;22(10):1247-54.

12. Koorstra JB, Maitra A, Morsink FH, Drillenburg P, ten Kate FJ, Hruban RH, et al. Undifferentiated carcinoma with osteoclastic giant cells (UCOCGC) of the pancreas associated with the familial atypical multiple mole melanoma syndrome (FAMMM). Am J Surg Pathol. 2008;32(12):1905-9. 
13. Imai Y, Morishita S, Ikeda Y, Toyoda M, Ashizawa T, Yamamoto K, et al. Immunohistochemical and molecular analysis of giant cell carcinoma of the pancreas: a report of three cases. Pancreas. 1999;18(3):308-15.

14. Luchini C, Pea A, Lionheart G, Mafficini A, Nottegar A, Veronese N, et al. Pancreatic undifferentiated carcinoma with osteoclast-like giant cells is genetically similar to, but clinically distinct from, conventional ductal adenocarcinoma. J Pathol. 2017;243(2):148-54.

15. Saito H, Kashiyama H, Murohashi T, Sasaki K, Misawa R, Ohwada S. Case of six-year disease-free survival with undifferentiated carcinoma of the pancreas. Case Rep Gastroenterol. 2016;10(2):472-8.

16. Sah SK, Li Y, Li Y. Undifferentiated carcinoma of the pancreas with osteoclast-like giant cells: a rare case report and review of the literature. Int J Clin Exp Pathol. 2015;8(9):11785-91.

17. Burkadze G, Turashvili G. A case of osteoclast-like giant cell tumor of the pancreas associated with borderline mucinous cystic neoplasm. Pathol Oncol Res. 2009;15(1):129-31

18. Kawamoto Y, Ome Y, Terada K, Hashida K, Kawamoto K, Ito T. Undifferentiated carcinoma with osteoclast-like giant cells of the ampullary region: short term survival after pancreaticoduodenectomy. Int J Surg Case Rep. 2016;24:199-202.

19. Bosman F, Carneiro F, Hruban RH, Theise ND. WHO classification of tumours of the digestive system. 4th ed. France: IARC Press; 2010.

20. Rosai J. Carcinoma of pancreas simulating giant cell tumor of bone. Electron-microscopic evidence of its acinar cell origin. Cancer. 1968;22(2): 333-44.

21. Baniel J, Konichezky M, Wolloch Y. Osteoclast-type giant cell tumor of the pancreas. Case report. Acta Chir Scand. 1987;153(1):67-9.

22. Fukushima N, Hruban RH, Kato Y, Klimstra DS, Kloppel G, Shimizu N, et al. Ductal adenocarcinoma variants and mixed neoplasms of the pancreas. In: Bosman FT, Carneiro F, Hruban RH, Theise ND, editors. WHO classification of tumors of the digestive system. Lyon: IARC; 2008. p. 292-9.

23. Sedivy R, Kalipciyan M, Mazal PR, Wolf B, Wrba F, Karner-Hanusch J, et al. Osteoclast-like giant cell tumor in mucinous cystadenocarcinoma of the pancreas: an immunohistochemical and molecular analysis. Cancer Detect Prev. 2005;29(1):8-14

24. Byth BC, Billingsley GD, Cox DW. Physical and genetic mapping of the serpin gene cluster at 14q32.1: allelic association and a unique haplotype associated with alpha 1-antitrypsin deficiency. Am J Hum Genet. 1994;55(1): 126-33.

25. Zhou J, Cheng Y, Tang L, Martinka M, Kalia S. Up-regulation of SERPINA3 correlates with high mortality of melanoma patients and increased migration and invasion of cancer cells. Oncotarget. 2017;8(12):18712-25.

26. Yang GD, Yang XM, Lu H, Ren Y, Ma MZ, Zhu LY, et al. SERPINA3 promotes endometrial cancer cells growth by regulating G2/M cell cycle checkpoint and apoptosis. Int J Clin Exp Pathol. 2014:7(4):1348-58.

27. Matissek SJ, Elsawa SF. GLI3: a mediator of genetic diseases, development and cancer. Cell Commun Signal. 2020;18(1):54.

28. Mueller S, Engleitner T, Maresch R, Zukowska M, Lange S, Kaltenbacher T, et al. Evolutionary routes and KRAS dosage define pancreatic cancer phenotypes. Nature. 2018;554(7690):62-8.

29. Jiang Y, Li Y, Zhu B. T-cell exhaustion in the tumor microenvironment. Cell Death Dis. 2015;6(6):e1792.

30. Wang L, Ma Q, Chen X, Guo K, Li J, Zhang M. Clinical significance of B7-H1 and B7-1 expressions in pancreatic carcinoma. World J Surg. 2010;34(5): $1059-65$.

31. Chen Y, Sun J, Zhao H, Zhu D, Zhi Q, Song S, et al. The coexpression and clinical significance of costimulatory molecules B7- $\mathrm{H} 1, \mathrm{~B} 7-\mathrm{H} 3$, and $\mathrm{B} 7-\mathrm{H} 4$ in human pancreatic cancer. Onco Targets Ther. 2014;7:1465-72.

32. Loos M, Giese NA, Kleeff J, Giese T, Gaida MM, Bergmann F, et al. Clinica significance and regulation of the costimulatory molecule $\mathrm{B} 7-\mathrm{H} 1$ in pancreatic cancer. Cancer Lett. 2008;268(1):98-109.

33. Geng L, Huang D, Liu J, Qian Y, Deng J, Li D, et al. B7-H1 up-regulated expression in human pancreatic carcinoma tissue associates with tumor progression. J Cancer Res Clin Oncol. 2008;134(9):1021-7.

34. Birnbaum DJ, Finetti $P$, Lopresti $A$, Gilabert M, Poizat F, Turrini $O$, et al. Prognostic value of PDL1 expression in pancreatic cancer. Oncotarget. 2016; 7(44):71198-210

35. Luchini C, Cros J, Pea A, Pilati C, Veronese N, Rusev B, et al. PD-1, PD-L1, and CD163 in pancreatic undifferentiated carcinoma with osteoclast-like giant cells: expression patterns and clinical implications. Hum Pathol. 2018;81:157-65.
36. Zhang CM, LV JF, Gong L, Yu LY, Chen XP, Zhou HH, et al. Role of deficient mismatch repair in the personalized management of colorectal cancer. Int Environ Res Public Health. 2016;13(9):892.

37. Le DT, Durham JN, Smith KN, Wang H, Bartlett BR, Aulakh LK, et al. Mismatch repair deficiency predicts response of solid tumors to PD-1 blockade. Science. 2017;357(6349):409-13.

38. Hu Zl, Shia J, Stadler ZK, Varghese AM, Capanu M, Salo-Mullen E, et al. Evaluating mismatch repair deficiency in pancreatic adenocarcinoma: challenges and recommendations. Clin Cancer Res. 2018;24(6):1326-36.

39. Kim ST, Klempner SJ, Park SH, Park JO, Park YS, Lim HY, et al. Correlating programmed death ligand 1 (PD-L1) expression, mismatch repair deficiency, and outcomes across tumor types: implications for immunotherapy. Oncotarget. 2017:8(44):77415-23.

40. Salem ME, Puccini A, Grothey A, Raghavan D, Goldberg RM, Xiu J, et al. Landscape of tumor mutation load, mismatch repair deficiency, and PD-L1 expression in a large patient cohort of gastrointestinal cancers. Mol Cancer Res. 2018;16(5):805-12.

41. Greer JB, Whitcomb DC. Role of BRCA1 and BRCA2 mutations in pancreatic cancer. Gut. 2007;56(5):601-5.

42. Luo G, Lu Y, Jin K, Cheng H, Guo M, Liu Z, et al. Pancreatic cancer: BRCA mutation and personalized treatment. Expert Rev Anticancer Ther. 2015; 15(10):1223-31.

43. Lowery MA, Kelsen DP, Stadler ZK, Yu KH, Janjigian YY, Ludwig E, et al. An emerging entity: pancreatic adenocarcinoma associated with a known BRCA mutation: clinical descriptors, treatment implications, and future directions. Oncologist. 2011:16(10):1397-402.

44. Lacour RA, Westin SN, Meyer LA, Wingo SN, Schorge JO, Brooks R, et al. Improved survival in non-Ashkenazi Jewish ovarian cancer patients with BRCA1 and BRCA2 gene mutations. Gynecol Oncol. 2011;121(2):358-63.

45. Kaufman B, Shapira-Frommer R, Schmutzler RK, Audeh MW, Friedlander M, Balmaña J, et al. Olaparib monotherapy in patients with advanced cancer and a germline BRCA1/2 mutation. J Clin Oncol. 2015;33(3):244-50.

46. Golan T, Hammel P, Reni M, Van Cutsem E, Macarulla T, Hall MJ, et al. Maintenance olaparib for germline BRCA-mutated metastatic pancreatic cancer. N Engl J Med. 2019;381(4):317-27.

47. Luo G, Long J, Zhang B, Liu C, Xu J, Ni Q, et al. Stroma and pancreatic ductal adenocarcinoma: an interaction loop. Biochim Biophys Acta. 2012; 1826(1):170-8

48. Conroy $T$, Desseigne $F, Y$ chou $M$, Bouché $O$, Guimbaud $R$, Bécouarn $Y$, et al. FOLFIRINOX versus gemcitabine for metastatic pancreatic cancer. N Engl J Med. 2011;364(19):1817-25.

49. Von Hoff DD, Ervin T, Arena FP, Chiorean EG, Infante J, Moore M, et al. Increased survival in pancreatic cancer with nab-paclitaxel plus gemcitabine. N Engl J Med. 2013:369(18):1691-703.

50. Kwa M, Edwards S, Downey A, Reich E, Wallach R, Curtin J, et al. Ovarian cancer in BRCA mutation carriers: improved outcome after intraperitoneal (IP) cisplatin. Ann Surg Oncol. 2014;21(5):1468-73.

51. Sakai W, Swisher EM, Karlan BY, Agarwal MK, Higgins J, Friedman C, et al. Secondary mutations as a mechanism of cisplatin resistance in BRCA2mutated cancers. Nature. 2008:451(7182):1116-20.

52. Golan T, Kanji ZS, Epelbaum R, Devaud N, Dagan E, Holter S, et al. Overall survival and clinical characteristics of pancreatic cancer in BRCA mutation carriers. Br J Cancer. 2014;111(6):1132-8.

53. Sehdev A, Gbolahan O, Hancock BA, Stanley M, Shahda S, Wan J, et al. Germline and somatic DNA damage repair gene mutations and overall survival in metastatic pancreatic adenocarcinoma patients treated with FOLFIRINOX. Clin Cancer Res. 2018;24(24):6204-11.

54. Tempero MA, Malafa MP, Chiorean EG, Czito B, Scaife C, Narang AK, et al. Pancreatic adenocarcinoma, version 1.2019. J Natl Compr Cancer Netw. 2019;17(3):202-10

55. Waddell N, Pajic M, Patch AM, Chang DK, Kassahn KS, Bailey P, et al. Whole genomes redefine the mutational landscape of pancreatic cancer. Nature. 2015;518(7540):495-501.

56. Wu C, Miao X, Huang L, Che X, Jiang G, Yu D, et al. Genome-wide association study identifies five loci associated with susceptibility to pancreatic cancer in Chinese populations. Nat Genet. 44(1):62-6.

\section{Publisher's Note}

Springer Nature remains neutral with regard to jurisdictional claims in published maps and institutional affiliations. 